\title{
Promoting Family Integrated Early Childhood Development During the First 1000 Days in Urban Slums of Delhi: Feasibility and Early Implementation Experience
}

\author{
Priyanka Khuda, Rifat Ali Fatima, Ayushi Bhatnagar, Mukesh Savaria, Manoja Kumar Das \\ From The INCLEN Trust International, Okhla Industrial Area, New Delhi. \\ Correspondence to: Dr Manoja Kumar Das, Director Projects, The INCLEN Trust International, F1/5, Okhla Industrial Area, \\ Phase 1, NewDelhi 110020.manoj@inclentrust.org
}

\begin{abstract}
The first 1000 days are critical for human development. Children residing in urban slums are at higher risk of suboptimal development. This article reports the feasibility and early implementation experiences of child stimulation directed counselling integrated with nutrition and health services by community functionaries in Delhi slums. This implementation research with cohort study design has two groups. The intervention group includes: pregnant women; infants and children in second year. The comparison group (without intervention) includes: recently delivered women, children aged 13-25 months. The feedback from the community functionaries and parents of children were also obtained. 44 videos with Hindi voiceover, demonstration of tools/toys/props, and 9 calendars depicting age-appropriate child stimulation activities were prepared and packaged with health and nutrition services for delivery by the frontline functionaries. These functionaries are coaching the families on child stimulation using these audio-visual messages using Tablets and demonstration tools/toys/props twice every three months. Monitoring and feedback data on service delivery are being collected. The data from the intervention cohort shall be compared with that from the comparison group to document the impact. From 63 anganwadi centres, 130 pregnant women, 142 infants and 136 children have been recruited for the intervention. $82.9 \%$ of the families received the intervention within 2 weeks and average time taken was 15 minutes. The initial feedback from the community functionaries and parents are very encouraging. The integrated child development package is acceptable and feasible for implementation through community functionaries and possible integration into the existing programs at scale.
\end{abstract}

Keywords: Child stimulation package, Frontline community functionaries.

Trial registration: CTRI/2019/01/016968

$\mathrm{T}$ he first 1000 days (conception through 24 months) is a critical period for development and future adult health. Poverty, deprived environments, poor sanitation and negative societal determinants challenge child developmental potential in the urban slums [1]. In metropolitan cities, 30$40 \%$ of population lives in slums or unorganized habitations [2]. The slum children suffer from repeated illnesses, malnutrition, and environments characterized by poor cognitive and social stimuli $[3,4]$.

The Integrated Child Development Services (ICDS) program of the Ministry of Women and Child Development (WCD) delivers nutrition, early child development (ECD) services, pre-school education and supportive health services through the anganwadi centers (AWCs) by the anganwadi workers (AWWs). The Ministry of Health and Family Welfare (MoHFW) also delivers health care, nutrition, and few ECD services in the community through the accredited social health activists (ASHAs) and auxiliary nurse midwives (ANM). These services from both ministries are largely complementary. In addition, the Rashtriya Bal Swasthya Karyakram targets detection of the birth defects, deficiencies, diseases and development delays including disability through periodic assessments.
The frontline service providers (AWWs and ASHAs) have inadequate skills and tools to counsel and demonstrate the ECD activities to parents [5-8]. The ASHAs/AWWs are likely to have similar challenges for ECD targeted service delivery and counselling. Without concrete demonstration of child stimulation techniques, parental practices and possible early detection of developmental delays by the field workers remain suboptimal. To assist parents practice child stimulation activities, the AWWs and ASHAs must be able to explain and demonstrate these effectively and appropriately. Audio-visual clips with tools demonstrating the targeted child stimulation activities can minimize the variations in counsell-ing by the providers, and also improve the understanding and longer retention of the parents [9].

This study aims to equip ASHAs, AWWs, and their supervisors with audio-visual and pictorial counselling messages that will enable them to demonstrate, educate and support parents, families and the local community in augmenting child development in Delhi urban slums. We hypothesize that counselling and demonstration of ageappropriate early child stimulation coupled with the health and nutrition messages for parents/family members in a sequential manner every quarter would improve parent's knowledge, practices, and child development status. This 
article describes the process of developing this early childhood stimulation intervention package and our early implementation experiences through the ASHAs and AWWs in urban slums of Delhi.

\section{METHODS}

The project has the following objectives: to develop an intervention package; to improve the early child development potential of the children in select urban slums of Delhi during first 1000 days by improving the parents/family knowledge and practices related to health, nutrition and child development; and to build capacity and enable ASHAs and AWWs for integrated delivery of health, nutrition and child development services.

This implementation research follows a cohort design. The protocol has been approved by the institutional ethics committee. The intervention groups include three parallel cohorts; pregnant women (recruited in the first trimester); infants (recruited in the first month of life and children (recruited at 13 month of age). These participants are contacted twice quarterly (ASHA and AWW separately at one month interval) for delivering the intervention package. The outcomes in the intervention groups shall be compared with three categories of participants not exposed to intervention from the same areas (comparison groups): recently delivered women (within last one month), children aged 13 months and children aged 25 months to document the differences. Perceptions and practices of the ASHAs, AWWs and family members are also being documented at periodic intervals.

Three urban slum areas from two districts of Delhi (Central Delhi and New Delhi) under the coverage of 63 AWCs (with ASHA and AWW) have been identified in consultation with State Government. These areas cover 134,400 population, who are catered by 68 AWWs and 34 ASHAs.

The project is being implemented in collaboration with State and District Health and WCD Authority. Ageappropriate audio-visual child stimulation modules that have been developed have been integrated into software program with nutrition, immunization and health compo-nents for service delivery by the ASHAs/AWWs. The ASHAs and AWWs have been trained (one day quarterly, prior to each round of intervention through demonstration and hands-on practice at respective primary health centres, provided with tablets, intervention packages for service delivery, and standard equipment for nutritional assessment. The participants have been identified with assistance from the ASHAs/ AWWs and recruited after obtaining informed consent by the research staff. The ASHAs/AWWs delivering the intervention using the tablets at the scheduled intervals are being notified by the software. Concurrent monitoring is being undertaken by documenting timeliness, completeness and quality of counselling and demonstration in addition to parental feedback. The ASHAs/AWWs are receiving performance based incentive as per the government guidelines. The imple-mentation of the project is reviewed by Senior Officials from Health and WCD departments.

The impact shall be assessed after one year of implementation for each cohort (pregnant women; infants and children). The outcome in the intervention groups shall be compared with the comparison groups (without intervention) from the same area to document the impact. The key impact indicators include: (1) For children: (1.1) knowledge and practice of the child stimulation activities by the families, (1.2) child development status (neuro-cognitive, motor, and language) at 13 month and 25 months of age as assessed by Bayley Scales of Infant and Toddler Development, 3rd edition (BSID III) and Developmental Assessment for Indian Infants (DASII), (1.3) nutritional status (weight, height, weight-forheight) and Infant and young child feeding practices and health status (immunization, frequency of illnesses and health care seeking behavior) of the children, and (2) For pregnant women: pregnancy and postpartum care practices. The data being collected by the trained project staff including child psycho-logists are summarized in Box I. The process indicators include visits made by ASHAs/AWWs (\%), visits completed within scheduled time (\%), data completeness (\%) and duration of contact (minutes). In-depth interviews (IDI) with the parents $(n=20)$ and ASHAs/AWWs $(n=10)$ are being conducted after each cycle to capture their perceptions, practices and experiences with the intervention.

The quantitative data are analyzed using descriptive statistics. The qualitative data are inductively analyzed following grounded theory and content analysis to identify domains following free listing, coding, axial coding and cross tabulation processes.

Intervention package development: Based on the literature review and available information, a team of paediatrician and psychologist compiled the age-appropriate child development milestones and early stimulation activities along with the tools/toys/props. For the child stimulation activities, audio-visual and pictorial messages featuring parent-child dyads from different age categories were prepared by professional filmmaker team. Hindi voiceover was done for these videos and parents and family members were consulted to ensure sociocultural appropriateness of scripts. In total 44 videos have been developed and organized under eight 3-monthly age bands. To remind and guide the parents/ family about age-appropriate child stimulation activities, eight calendars corresponding to the three-monthly age bands for the first two years of age were compiled. These calendars have three colour-coded sections with pictorial 
Pregnant Women

Woman-during pregnancy: antenatal care, dietary practices, nutrition supplements, physical activity, quality of life, depression, anxiety, stress, addictions, domestic violence, and social support, awareness about danger signs during pregnancy, problems and complications;

Woman-after termination/delivery: pregnancy outcome, delivery care, postpartum period and danger signs;

Newborn (after delivery): birthweight, breastfeeding, awareness about danger signs and immunization in newborn/infant.

Infants

Infant/Child (at recruitment and after one year): ${ }^{a}$ breastfeeding, complementary feeding, dietary diversity, immunization, anthropometry (weight, length and weight-for-height/length), illnesses and care seeking during illnesses, development status (motor, sensory, language and cognitive) using BSID III/DASII scale;

Mother/caretaker (at recruitment and after one year): ${ }^{b}$ knowledge about the child development stimulus practices, awareness about the development delays, childhood illnesses, care seeking, quality of life, depression, anxiety, stress, addictions, domestic violence, social support, home environment.

All participants

Family composition, socio-economic status, sanitation and hygiene practice.

Process indicators

Contact schedule and timeliness, feedback from the ASHAs/AWWs, feedback from mothers.

In comparison group, ${ }^{a}$ the child and the ${ }^{b}$ mother/care taker will be assesed only once. ASHA-Accredited Social Health Activist; AWW-Anganwadi worker; BSID-Bayley Scales of Infant and Toddler Development; DASII- Developmental Assessment Scales for Indian Infants.

messages: $a$ ) what the child is expected to do/achieve during these months; $b$ ) what child stimulation activities parents should do; and $c$ ) the features of developmental delay (red flags). Additionally, a pictorial calendar for pregnancy care have been developed with three sections: $a$ ) what pregnant woman should do; $b$ ) what pregnant woman should not do; and $c$ ) the danger signs for seeking care. (Suppl. Document 1). Eight packets of age- and activity-appropriate tools/ toys/props (used in the videos/pictures) have been compiled to demonstrate the age appropriate stimulation activities. The audio-visual messages, calendars and tools/ toys/props have been pre-tested with 10 mothers with children from each age-band $(n=80)$ and 20 pregnant women from two areas for comprehension, clarity and socio-cultural appropriateness, and refined accordingly. The framework and sequence of intervention package delivery timings are shown in Web Fig. 1.

Software package for implementation: The dedicated software program developed for service delivery by ASHAs/ AWWs has two parts: android user application and web application (admin management and analytics). For children, the application enables recording anthropometry, immunization, feeding, illness/danger signs, milestones achieved and development delays during each contact. The pregnant women component integrates the trimester-appropriate nutrition, pregnancy care, immunization and danger signs for counse-lling. After data capture, the ASHA/AWW shows the videos and explains the calendar. The contact duration and videos viewing times are auto-recorded (Suppl. Document 2).

\section{RESULTS}

First attempt: We initiated participant recruitment during February-March 2020 and recruited 59 pregnant women, 90 infants and 80 children. But, due to the COVID-19 pandemic lockdown and restrictions, the training of ASHAs/AWWs and field activities were suspended. No implementation by the ASHAs/AWWs could be initiated. Due to these unexpec-ted challenges, we considered virtual counselling for the recruited families as an exploratory implementation. The research staff could contact parents of 65 infants and 38 children. These parents were shared the videos and screenshots through Whatsapp and counselled through visioncall at scheduled intervals. All four quarterly counselling packages have been delivered virtually to these parents. The data collection after one year of intervention is under-way. The average times taken for package delivery were; 15 minutes (cycle-1), 16 minutes (cycle-2), 14 minutes (cycle-3) and 15 minutes (cycle -4 ).

Second attempt: Following the cessation of the lockdown, 130 pregnant women, 142 infants and 136 children have been recruited in the intervention groups during NovemberDecember 2020. Comparison groups have been recruited as described earlier. Following the training, the first and second intervention packages have been delivered to all the intervention group participants by the ASHAs/AWWs and third cycle is under progress. The process indicators for the first two cycles are summarized in Table I. The aforementioned study specific have been captured.

Experience of ASHAs/AWWs and parents: Data saturation for the IDIs with the ASHAs/AWWs and parents were reached with 17-18 IDIs. The feedbacks from ASHAs/ AWWs after two intervention cycles were encouraging. The ASHAs/AWWs found the issue quite relevant to the overall mother and child care package. Their knowledge and skills related to child development improved after the training and gained confidence for counselling the parents. 
The mothers found the videos and demonstration of how to use the tools/toys/props very useful. Several parents informed that they have been doing the activities as shown in the video and calendar by the ASHAs/AWWs. Some statements from the ASHAs/AWWs and mothers after first two intervention cycles are summarized in Web BoxI.

Impact assessment: The impact assessment shall be done after delivery of four intervention packages, when the pregnant women deliver, infants become 13 months old and children become 25 months old.

\section{DISCUSSION}

The first 1000 days has attracted attention of clinicians and maternal and child programs from government and nongovernment initiatives. In absence of standardized socioculturally appropriate messages and demonstration tools for the frontline functionaries, the service delivery is challenged and lacks uniformity. Counselling and demonstration of activities depends largely on the competence and skill of the functionaries. The intervention packages are being delivered to the families from poor socioeconomic status in the slum areas, who have poor literacy levels and limited access to information. The children in slums are exposed to multitude of adversities. The negative impact of these adversities may be minimised with early child intervention packages that include stimulation activities coupled with counselling directed at improving diets, care during illnesses and vaccination practices. Early initiation of the child development targeted counselling from the first month of life and repeated contacts are likely to allow sufficient opportunity and time for practice transformation by parents, as documented in some interventions [10].

Use of videos and demonstrating toys/tools/props by ASHAs/AWWs while counselling parents are likely to ensure uniformity and minimize variation in communication. The age-appropriate pictorial calendar assists parents in continued practice of child stimulation activities. The implementation by ASHAs/AWWs integrated with the maternal and child health program provides opportunity for documenting the operational aspects and lessons for potential adoption within the ongoing program. The exploratory virtual ECD package delivery during the COVID-19 lockdown documents the feasibility of implementation during the pandemic/disaster situations, when physical contact is difficult.

The initial feedback from ASHAs/AWWs indicate improvements in their knowledge, skill and confidence related to child development counselling. The provision of performance linked incentives is motivating them. Monitoring of timeliness, complete package delivery by ASHAs/ AWWs and data captured using the software installed tablets are emerging as key quality assurance measures. Delivery of the interventions through ASHAs/AWWs and involvement of the health and WCD systems across all levels ensures feasibility and the alignment with program and policy. There is improvement in knowledge and practice of parents regarding the child development as counselled by ASHAs/AWWs. Integrated delivery of child stimulation activities along with nutrition and health messages improve the chances of acceptance and sustenance, as observed in several studies [11,12].

This study has some limitations. This manuscript presents the early experiences of implementation and impact results are pending. The shorter time period of implementation is a limitation.

There is no doubt regarding the role of social-play and child stimulation activities by parents and family during first 1000 days on child's development. The early experiences demonstrate the feasibility of implementing the integrated child stimulation package using tablets by the frontline functionaries in urban slums. Using the audio-visual communi- cation and demonstrating toys/tools/props improves the comprehension and increases the chance of adoption by the family. The impact of such intervention package and process documentation shall inform government and stakeholders for potential adoption and scaling up within the existing program.

Table I Process Indicators for the Intervention Delivery to the Beneficiary Families (During First and Second Intervention Cycles)

\begin{tabular}{llllr}
\hline Beneficiary category & \multicolumn{2}{c}{ Contacts } & \multicolumn{2}{c}{ Time taken for intervention delivery $\left(\right.$ min) $^{a}$} \\
\cline { 2 - 3 } \cline { 5 - 5 } & Withinfirstweek $(\%)$ & Within secondweek (\%) & Firstcycle & Second cycle \\
\hline Pregnant women & 56.9 & 83.8 & $14.4(13.4-16.5)$ & $14.3(13.5-15.5)$ \\
Infants & 59.6 & 83.0 & $15.4(14.0-16.5)$ & $15.3(15.0-16.5)$ \\
Children & 59.4 & 82.0 & $16.0(14.2-17.5)$ & $15.4(14.1-16.4)$ \\
Pooled & 58.6 & 82.9 & $15.2(14.1-16.4)$ & $15.0(14.0-16.1)$ \\
\hline
\end{tabular}

${ }^{a}$ Data in median (IQR). 
Ethics clearance: INCLEN Ethics Committee; No. IIEC-51 dated July 5, 2018.

Note: Supplementary material related to this manuscript is available at $w w w$. indianpediatrics.net

Acknowledgements: Ms Madhumati Bose, Ms Savita Sapra and Dr Surender Bisht, contributed to the development of the audiovideo and pictorial contents and tools for early child development. We thank the Departments of Health and Family Welfare and Women and Child Development, Government of NCT of Delhi for facilitating the implementation of the project in the districts. We highly appreciate the facilitation and support from the District Health Administration and ICDS Program Administration and the functionaries in the districts of Central Delhi and New Delhi for implementing the project activities.

Contributors: PK, RAF, AB, MS: intervention package development, data collation, data analysis, manuscript review and final approval of the manuscript; MKD: conceptualisation, intervention package development, data collation, data analysis, manuscript writing, final approval of the manuscript.

Funding: Grand Challenges Canada (Grant No.SB-POC-181020754) under the program Contribution in support of Grand Challenges Canada's Innovation Platform for Maternal, Newborn and Child Health (MNCH). Competing interest: None stated.

\section{REFERENCES}

1. Unger A. Children's health in slum settings. Arch Dis Child. 2013;98:799-805.

2. United Nations, Department of Economic and Social Affairs. Population Division (2018). World Urbanization Prospects: The 2018 Revision [Internet]. United Nations; 2018. Accessed July 2, 2019. Available from: https://population.un.org/wup/ Publications/Files/WUP2018-Report.pdf

3. Kavitha N. Are slum children at high risk of under nutrition, anemia and childhood morbidity? Evidence from India. Ind J
Comm Health. 2014;26:124-31.

4. Sunitha S, Gururaj G. Health behaviours \& problems among young people in India: Cause for concern \& call for action. Indian J Med Res. 2014;140:185-208.

5. Chaturvedi A, Nakkeeran N, Doshi M, Patel R, Bhagwat S. Capacity of frontline ICDS functionaries to support caregivers on infant and young child feeding (IYCF) practices in Gujarat, India. Asia Pac J Clin Nutr. 2014;23:S29-37.

6. Kohli C, Kishore J, Sharma S, Nayak H. Knowledge and practice of Accredited Social Health Activists for maternal healthcare delivery in Delhi. J Family Med Prim Care 2015;4:359-63.

7. Kohli S, Chadha R. Knowledge and counselling skills of community health workers for promotion of optimal Infant and Young Child Feeding (IYCF) practices: a review. Int J Health Sci Res. 2017;7:240-51.

8. Smittenaar P, Ramesh BM, Jain M, et al. Bringing greater precision to interactions between community health workers and households to improve maternal and newborn health outcomes in India. Glob Health Sci Pract. 2020;8:358-71.

9. Adam M, McMahon SA, Prober C, Bärnighausen T. Humancentered design of video-based health education: An iterative, collaborative, community-based approach. J Med Internet Res. 2019; 21:e12128.

10. Roia A, Paviotti E, Ferluga V, et al. Promoting effective child development practices in the first year of life: does timing make a difference? BMC Pediatr. 2014;14:222.

11. Yousafzai AK, Rasheed MA, Rizvi A, et al. Effect of integrated responsive stimulation and nutrition interventions in the Lady Health Worker programme in Pakistan on child development, growth, and health outcomes: A cluster-randomised factorial effectiveness trial. Lancet. 2014;384:1282-93.

12. Yousafzai AK, Obradoviæ J, Rasheed MA, et al. Effects of responsive stimulation and nutrition interventions on children's development and growth at age 4 years in a disadvantaged population in Pakistan: A longitudinal follow-up of a clusterrandomised factorial effectiveness trial. Lancet Glob Health. 2016;4:e548-58. 